\title{
Características de la Fibra de Alpaca Huacaya de Cotaruse, Apurímac, Perú
}

\author{
Fiber Characteristics of Huacaya Alpaca at Cotaruse, Apurímac, Perú \\ V. Machaca Machaca ${ }^{1}$, A.V. Bustinza Choque ${ }^{2,6}$, F.A. Corredor Arizapana ${ }^{3}$, \\ V. Paucara Ocsa ${ }^{1}$, E.E. Quispe Peña ${ }^{4}$, R. Machaca Machaca ${ }^{5}$
}

\section{Resumen}

El objetivo del estudio fue establecer el perfil de las principales características físicas de la fibra de alpaca que pueden servir para su mejor comercialización y para fines de mejoramiento genético. Se hicieron mediciones de 145 muestras de colores blanco, intermedio y oscuro pertenecientes a alpacas de cinco comunidades del distrito de Cotaruse, Apurímac, Perú, utilizando el equipo ODFA2000 para determinar el diámetro de fibra (DF), el coeficiente de variación (CV[DF]), el factor de confort (FC) y el índice de curvatura (IC). Se estimaron los efectos de la comunidad, el sexo, edad, color de la fibra y sitio de muestreo sobre el DF, CV(DF), FC e IC y la relación entre ellos. El promedio del diámetro de fibra (MDF) estuvo influenciado por la edad ( $\mathrm{p}<0.01$ ) (valores entre 21.61 y $24.32 \mu \mathrm{m}$ ), por color de fibra $(22.30,23.81$ y $26.69 \mu \mathrm{m}$ para blanco, intermedio y oscuro, respectivamente) y por comunidad (de $21.9 \mu \mathrm{m}$ para Iscahuaca a $24.2 \mu \mathrm{m}$ para San Miguel de Mestizas) y por sexo $(\mathrm{p}<0.05)$, siendo la fibra de las hembras $1 \mu \mathrm{m}$ más fina que la de los machos; sin embargo, no hubo diferencias significativas por la zona corporal de la toma de la muestra. El CV(DF) mostró diferencias significativas por efecto de la edad $(\mathrm{p}<0.01)$ y por sexo y sitio de muestreo $(\mathrm{p}<0.05)$, pero sin diferencias por color y comunidad. El FC tuvo diferencias significativas $(\mathrm{p}<0.01$ ) por efecto de la comunidad y color de la fibra, así como por edad, sexo y sitio de muestreo $(\mathrm{p}<0.05)$. El IC tuvo diferencias significativas debido a la comunidad $(\mathrm{p}<0.01)$, edad, sexo y color $(\mathrm{p}<0.05)$, pero no por el sitio de muestreo. La MDF presentó una alta y negativa correlación con FC $(\mathrm{r}=-0.99)$ e IC $(\mathrm{r}=-0.61)$ y la FC presentó una correlación positiva con $\mathrm{IC}(\mathrm{r}=0.62)$. No se encontró una definición clara del sitio apropiado para el muestreo del vellón de alpaca, pero se puede realizar a la

1 Facultad de Medicina Veterinaria y Zootecnia, Universidad Nacional Micaela Bastidas, Apurímac, Perú

${ }^{2}$ Facultad de Medicina Veterinaria y Zootecnia, Universidad Nacional del Altiplano, Puno, Perú

${ }^{3}$ Programa de Cría de Animales, Universidad Nacional Agraria La Molina - UNALM, Lima, Perú

${ }^{4}$ Vicerrectoría de Investigación, Universidad Autónoma Nacional de Chota, Cajamarca, Perú

${ }^{5}$ Instituto Superior Tecnológico Público de Sangarara, Cusco, Perú

${ }^{6}$ E-mail:vicbust@hotmail.com

Recibido: 21 de abril de 2017

Aceptado para publicación: 8 de agosto de 2017 
edad de 1 año. Las alpacas del distrito de Cotaruse, Apurímac, indistintamente del color del vellón, producen una buena calidad de fibra y hay un gran potencial de variabilidad para su mejoramiento genético.

Palabras clave: alpaca; Huacaya; características de fibra; sexo; edad; comunidad de color

\section{Abstract}

The objective of the study was to establish the profile of the main physical characteristics of the alpaca fibre that can serve for a better commercialization and for breeding purposes. Measurements were made of 145 white and coloured fibre samples (brown and dark shaded) from five communities of the district of Cotaruse, Apurímac, Peru, using the ODFA 2000 equipment to determine fibre diameter (DF), coefficient of variation $(\mathrm{CV}[\mathrm{DF}])$, comfort factor $(\mathrm{FC})$ and curvature index $(\mathrm{CI})$. The effects of community, sex, age, fibre colour and body area of sampling on DF, CV(DF), FC and IC and their relationships were estimated. The mean fibre diameter (MDF) was affected by age $(\mathrm{p}<0.01)$ (values between 21.61 and $24.32 \mu \mathrm{m}$ ), by fibre colour (22.30,23.81 and $26.69 \mu \mathrm{m}$ for white, brown shaded and dark shaded, respectively) and by community ( $21.9 \mu \mathrm{m}$ for Iscahuaca and $24.2 \mu \mathrm{m}$ for San Miguel de Mestizas) and by sex $(\mathrm{p}<0.05)$, where the fibre in females were 1 ìm thinner than in males. However, there were no significant differences in the body area of sampling site. The CV(DF) showed significant differences due to age $(\mathrm{p}<0.01)$ and by sex and body area $(\mathrm{p}<0.05)$, but without differences by colour and community. The $\mathrm{CF}$ had significant differences $(\mathrm{p}<0.01)$ due to community and fibre colour, as well as by age, sex, and body area $(\mathrm{p}<0.05)$. The $\mathrm{FC}$ had significant differences due to the community $(\mathrm{p}<0.01)$, age, sex, and colour $(\mathrm{p}<0.05)$, but not by the sampling site. The MDF presented a high and negative correlation with $\mathrm{FC}(\mathrm{r}=-0.99)$ and $\mathrm{FC}(\mathrm{r}=-0.61)$ and $\mathrm{FC}$ had a positive correlation with IC $(\mathrm{r}=0.62)$. There was no clear definition of the appropriate sampling site, but it can be done at the age of 1 year. The alpacas of the district of Cotaruse, Apurímac, regardless of the colour of the fleece, produce a good quality of fibre and there is a great potential of variability for its genetic improvement.

Key words: alpaca; Huacaya; fibre characteristics; sex; age; colour; community

\section{INTRODUCCIÓN}

El Perú tiene una población de 3685516 de alpacas (INEI, 2012), correspondiendo el 5.9\% (219 113 alpacas) a Apurímac. La ganadería alpaquera es una actividad de gran importancia para una población que habita a más de 4000 metros sobre el nivel del mar y que, además, presenta las mayores tasas de pobreza (Germaná et al., 2016).

En Apurímac y en todo el Perú, la cría de alpacas se realiza, entre otras, para la pro- ducción de fibra para los mercados nacional y mundial y para la producción de carne para el mercado nacional (Bustinza, 2001). La fibra grasienta se vende a la industria peruana, pero la transformada para tops y prendas de vestir se exportan a los principales mercados de Italia, Estados Unidos, China, Inglaterra, Alemania, etc. (Mellisho, $\mathrm{s} / \mathrm{f}$ ).

En Apurímac, la crianza de alpacas se lleva a cabo principalmente en las provincias de Antabamba y de Aymaraes. Las comunidades del distrito de Cotaruse, consideradas en el estudio, son las más importantes en esta 
Cuadro 1. Alpacas muestreadas por comunidades del distrito de Cotaruse, Apurímac (Perú), de acuerdo con el sexo, color de la capa y edad

\begin{tabular}{lcccccccccc}
\hline \multirow{2}{*}{ Localidad } & \multicolumn{3}{c}{ Sexo } & \multicolumn{4}{c}{ Edades (años) } & \multicolumn{3}{c}{ Colores } \\
\cline { 2 - 9 } & $\mathrm{M}$ & $\mathrm{H}$ & 1 & 2 & 3 & 4 & $\mathrm{~B}$ & $\mathrm{INT}$ & OSC & \\
\hline Iscahuaca & 10 & 20 & 9 & 6 & 9 & 6 & 25 & 3 & 2 & 30 \\
Pampamarca & 6 & 1 & 0 & 1 & 2 & 4 & 3 & 3 & 1 & 7 \\
Pilluni & 24 & 18 & 7 & 9 & 11 & 15 & 24 & 10 & 8 & 42 \\
Quillcaccasa & 0 & 30 & 7 & 6 & 5 & 12 & 25 & 4 & 1 & 30 \\
SM Mestizas & 12 & 24 & 15 & 7 & 6 & 8 & 23 & 9 & 4 & 36 \\
\hline Total & 52 & 93 & 38 & 29 & 33 & 45 & 100 & 29 & 16 & 145 \\
\hline
\end{tabular}

$\mathrm{M}=$ machos, $\mathrm{H}=$ hembras, $\mathrm{B}=$ blanco, $\mathrm{INT}=$ intermedio, $\mathrm{OSC}=$ colores oscuros

última provincia. A pesar de su importancia socioeconómica, poco se conoce sobre la calidad de la fibra producida en estas áreas, aunque se han hecho esfuerzos para mejorar la calidad de la fibra con la introducción de reproductores de las poblaciones de Puno y Cusco (Vásquez et al., 2015).

El presente estudio tuvo como objetivo determinar las características físicas naturales de alpacas pertenecientes a cinco comunidades con tradición en crianza de alpacas en el distrito de Cotaruse, cuyos resultados servirán como un elemento importante de apoyo a los productores para una mejor negociación en el momento de la comercialización de la fibra, a nivel local o internacional, y también para el desarrollo de programas de mejoramiento genético para lograr la producción de fibra que podría satisfacer las necesidades del mercado textil.

\section{Materiales Y MéTodos}

Se trabajó con la fibra proveniente de 145 alpacas pertenecientes a cinco comunidades (Iscahuaca, Pampamarca, Pilluni, Quilcaccasa y San Miguel de Mestizas) del distrito de Cotaruse, provincia de Aymaraes, región de Apurímac, ubicada en la parte sur del Perú. Las comunidades se localizan entre 4100 y 4800 msnm y se caracterizan por presentar pastos naturales de vegetación herbácea con predominio de gramíneas, leguminosas y plantas de las familias Cyperaceae, Rosaceae, Compositaceae, Malvaceae y Geraniaceae, entre otras (Tapia y Flores, 1984).

Las muestras de fibra se tomaron en alpacas con color de capa entera, que fueron clasificadas como blanco, intermedio (colores claros o light fawns) y oscuro (marrón, gris y negro). Las muestras de fibra, en cantidad de $10 \mathrm{~g}$, fueron tomadas de la paleta, costillar medio y grupa. No se consideraron las alpacas con defectos genéticos como vellón manchado, orejas cortas, prognatismo inferior o superior y ojos zarcos. La edad se determinó mediante la evaluación de los dientes (dientes de leche, dos dientes, cuatro dientes y boca llena, que equivalen a 1, 2, 3 y 4 años o más de edad) (Cuadro 1).

El muestreo se llevó a cabo en abril de 2015, antes de que los animales fueran esquilados. Las muestras fueron colocadas en bolsas de polietileno rotuladas con el nombre del propietario, comunidad, color, sexo y fecha de muestreo. 
Se determinaron las siguientes mediciones de fibra: diámetro de la fibra (DF), coeficiente de variación del diámetro de la fibra (CV[DF]), factor de confort (FC) e índice de curvatura (IC). Estas mediciones se llevaron a cabo en el laboratorio de fibras de la Universidad Nacional de Huancavelica (Huancavelica, Perú), utilizando el ODFA 2000 (Analizador de Diámetro de Fibra Óptico), reconocido por IWTO (Organización Internacional de Textiles de Lana, TM 47).

Los efectos de la comunidad, edad, sexo, color de la fibra y sitio de muestreo sobre las variables en estudio se calcularon utilizando la aplicación R v. 3.0.2 (R Core Team, 2014).

\section{Resultados y Discusión}

La media ( \pm error estándar) del diámetro de fibra (MDF) fue de $23.09 \pm 0.31 \mu \mathrm{m}$ (Cuadro 2), con un rango de 15.10 a $31.53 \mu \mathrm{m}$. Un estudio reciente en la comunidad de Iscahuaca, distrito de Cotaruse, reportó un valor menor $(19 . \pm 0.2 \mu \mathrm{m})$, con diferencias de $3.19 \mu \mathrm{m}$ entre fibras procedentes de alpacas de 1 a 4 años (Vásquez et al., 2015). Valores similares fueron reportados en estudios realizados en áreas cercanas a Cotaruse: $22.7 \pm 0.2 \mu \mathrm{m}$ (Montes et al., 2008) y $20.94 \mu \mathrm{m}$ (Quispe, 2010), ambos en Huancavelica, y de $22.78 \mu \mathrm{m}$ (Huanca et al., 2007) y $20.98 \pm$ $0.36 \mu \mathrm{m}$ (Ormachea, 2015), ambos en Carabaya, Puno. En alpacas criadas en Nueva Zelanda, McGregor y Butler (2004) encontraron una MDF de $29.1 \mu \mathrm{m}$ (17.7-46.6) y Lupton et al. (2006) en alpacas criadas en EEUU reportaron una MDF de $27.85 \mu \mathrm{m}$, con un rango de 15.09 a $49.27 \mu \mathrm{m}$, que resultan ser valores superiores a los encontrados en este estudio, probablemente debido a un mejor nivel de nutrición y de manejo.

El CV general del DF fue relativamente alto, con un valor de $22.59 \pm 0.18 \%$ (17.83$28.27 \%$ ), lo que implica que las alpacas de Cotaruse tienen una variación significativa en el diámetro de la fibra. El FC fue alto, con un valor de $87.12 \pm 1.02 \%$ (variando entre $48 \mathrm{y}$ $100 \%$ ) y el IC fue de $36.63 \pm 0.76 \% \mathrm{~mm}$ (variando entre 12.83 y $59.37 \% \mathrm{~mm}$ ), lo que indica que esta fibra tiene una característica de buen confort en la ropa de vestir, aunque la curvatura de la fibra es relativamente baja.

El FC y el IC estuvieron moderadamente correlacionados $(r=0.62)$, lo que indica que hay correspondencia entre ellos dentro de cada edad. También se observaron dos correlaciones negativas de MDF con IC ( $\mathrm{r}=-0.61)$ y MDF con FC ( $\mathrm{r}=-0.99)$, lo que indica que la menor finura de la fibra es menos cómoda y tiene menos ondulaciones (Cuadro 3).

El Estado Peruano ha estandarizado la fibra de alpaca por grupos de calidad y requerimientos textiles (Norma Técnica Peruana N. ${ }^{\circ}$ 231.301: 2004; Gutiérrez, 2011). Los resultados de la evaluación de las fibras en estudio, de acuerdo con esta norma, se presentan en el Cuadro 4. Por otro lado, si se juntan los grupos de fibras con menos de $30 \mu \mathrm{m}$ (límite de factor confort), alcanza al 91.3\%. Como referencia comparativa, McGregor (2006) indicó que alrededor del 10\% de las alpacas Huacaya tenían vellones con un MDF menor de $24.0 \mu \mathrm{m}$, mientras que el $14 \%$ de Suris tenían vellones menores de $24 \mu \mathrm{m}$, y que tanto Huacayas como Suris tenían alrededor del 50\% de vellones con una MDF de más de $29.9 \mu \mathrm{m}$.

Este conjunto de valores indicaría que las alpacas de Cotaruse, y muy probablemente de las zonas aledañas del sur del Perú, estarían produciendo fibras con buena finura para los requerimientos de la industria textil y, por otra parte, las fibras de alpacas de Nueva Zelanda y los Estados Unidos de América tendrían valores más altos de MDF.

\section{Efecto de la Edad}

La edad tiene un impacto muy fuerte en el diámetro de la fibra (MDF), ya que a mayor edad aumenta esta medida $(\mathrm{p}<0.01)$. $\mathrm{Si}$ se toma como referencia la MDF al año, la diferencia fue de $1.41,2.26$ y $2.71 \mu \mathrm{m}$ más 
Cuadro 2. Media del diámetro de la fibra (MDF), coeficiente de variación del diámetro de la fibra (CV[DF]), factor de confort (FC) e índice de curvatura (IC) de fibra de alpaca de las comunidades del distrito de Cotaruse, Apurímac (Perú), según la comunidad, edad, sexo, color de fibra y sitio de muestreo

\begin{tabular}{|c|c|c|c|c|c|}
\hline Variable & $\mathrm{N}$ & $\mathrm{MDF} \pm$ e.e. & $\mathrm{CV}(\mathrm{DF}) \pm$ e.e. & $\mathrm{FC} \pm$ e.e. & $\mathrm{IC} \pm$ e.e. \\
\hline Edad (años) ${ }^{1}$ & & $* * *$ & $* * *$ & $* *$ & $* *$ \\
\hline 1 (DL) & 38 & $21.61^{\mathrm{b}} \pm 0.65$ & $23.68^{\mathrm{a}} \pm 0.35$ & $92.38^{a} \pm 4.42$ & $33.35^{\mathrm{c}} \pm 1.31$ \\
\hline $2(2 \mathrm{D})$ & 29 & $22.22^{\mathrm{b}} \pm 0.63$ & $23.04^{\mathrm{ab}} \pm 0.39$ & $92.02^{\mathrm{ab}} \pm 4.14$ & $40.19^{\mathrm{a}} \pm 1.43$ \\
\hline 3 (4D) & 33 & $23.87^{\mathrm{a}} \pm 0.71$ & $21.46^{\mathrm{c}} \pm 0.34$ & $88.13^{\mathrm{bc}} \pm 4.88$ & $38.60^{\mathrm{ab}} \pm 1.61$ \\
\hline$>3(\mathrm{BLL})$ & 45 & $24.32^{\mathrm{a}} \pm 0.46$ & $22.22^{\mathrm{bc}} \pm 0.30$ & $86.45^{\mathrm{c}} \pm 3.21$ & $35.66^{\mathrm{bc}} \pm 1.50$ \\
\hline Sexo & & $*$ & $*$ & $*$ & $* *$ \\
\hline Macho & 52 & $23.79^{\mathrm{a}} \pm 0.50$ & $23.13^{\mathrm{a}} \pm 0.32$ & $87.41^{\mathrm{b}} \pm 3.39$ & $33.76^{\mathrm{b}} \pm 1.13$ \\
\hline Hembra & 93 & $22.79^{b} \pm 0.40$ & $22.30^{\mathrm{b}} \pm 0.21$ & $91.23^{\mathrm{a}} \pm 2.66$ & $38.23^{\mathrm{a}} \pm 0.97$ \\
\hline Localidad & & $* * *$ & NS & $* * *$ & $* * *$ \\
\hline Iscahuaca & 30 & $21.9^{\mathrm{bc}} \pm 0.62$ & $22.39^{\mathrm{ab}} \pm 0.46$ & $92.98^{\mathrm{ab}} \pm 4.13$ & $36.97^{\mathrm{b}} \pm 1.31$ \\
\hline Pampamarca & 7 & $24.5^{\mathrm{a}} \pm 0.45$ & $21.33^{b} \pm 0.97$ & $85.97^{\mathrm{c}} \pm 4.73$ & $31.43^{\mathrm{b}} \pm 4.01$ \\
\hline Pilluni & 42 & $23.5^{\mathrm{ab}} \pm 0.61$ & $22.46^{\mathrm{ab}} \pm 0.31$ & $87.32^{\mathrm{bc}} \pm 4.18$ & $35.38^{\mathrm{b}} \pm 1.57$ \\
\hline Quillcaccasa & 30 & $23.2^{\mathrm{c}} \pm 0.54$ & $22.66^{\mathrm{ab}} \pm 0.34$ & $95.86^{\mathrm{a}} \pm 3.36$ & $43.07^{\mathrm{a}} \pm 1.34$ \\
\hline SM Mestizas & 36 & $24.2^{\mathrm{a}} \pm 0.61$ & $23.11^{\mathrm{a}} \pm 0.38$ & $84.20^{\mathrm{c}} \pm 4.10$ & $33.46^{\mathrm{b}} \pm 1.36$ \\
\hline Color & & $* * *$ & NS & $* * *$ & $* *$ \\
\hline Blanco & 100 & $22.30^{b} \pm 0.34$ & $22.66^{\mathrm{a}} \pm 0.21$ & $92.16^{\mathrm{a}} \pm 2.30$ & $38.29^{\mathrm{a}} \pm 0.90$ \\
\hline Intermedio & 29 & $23.81^{\mathrm{b}} \pm 0.66$ & $22.41^{\mathrm{a}} \pm 0.46$ & $87.89^{\mathrm{a}} \pm 4.54$ & $34.98^{\mathrm{a}} \pm 1.39$ \\
\hline Oscuro & 16 & $26.69^{\mathrm{a}} \pm 0.97$ & $22.50^{\mathrm{a}} \pm 0.48$ & $75.94^{\mathrm{b}} \pm 6.62$ & $29.26^{\mathrm{b}} \pm 2.41$ \\
\hline Sitio de muestreo & & NS & $* *$ & $*$ & NS \\
\hline Costillar & 145 & $22.90^{\mathrm{a}} \pm 0.33$ & $22.20^{c} \pm 0.19$ & $91.21^{\mathrm{a}} \pm 2.21$ & $36.37^{\mathrm{a}} \pm 0.79$ \\
\hline Grupa & 145 & $23.01^{\mathrm{a}} \pm 0.35$ & $22.61^{\mathrm{ab}} \pm 0.20$ & $90.62^{\mathrm{ab}} \pm 2.37$ & $37.49^{\mathrm{a}} \pm 0.78$ \\
\hline Paleta & 145 & $23.71^{\mathrm{a}} \pm 0.36$ & $23.02^{\mathrm{a}} \pm 0.21$ & $88.32^{\mathrm{b}} \pm 2.48$ & $35.70^{\mathrm{a}} \pm 0.83$ \\
\hline Media General & 145 & $23.09 \pm 0.31$ & $22.59 \pm 0.18$ & $87.12 \pm 1.02$ & $36.63 \pm 0.76$ \\
\hline
\end{tabular}

fina que la MDF a los 2, 3 y $\geq 4$ años, respectivamente. Estos valores contrastan con el estudio de Vásquez et al. (2015) para la comunidad de Iscahuaca, donde las diferencias fueron más amplias; así la MDF al año (17.8) se diferenciaba en $1.9,2.9$ y $4.3 \mu \mathrm{m}$ de las edades de 2 y 4 años, respectivamente. Estos datos coinciden, en sentido general, con los datos presentados en los informes de
Montes et al. (2008) en Huancavelica y Ormachea et al. (2015) en Carabaya, Puno, y con los datos encontrados en EEUU por Lupton et al. (2006). Asimismo, Wuliji et al. (2000) reportaron valores de diámetro de fibra de $31.9 \pm 0.5,30.5 \pm 0.9$ y $26.44 \pm 0.4 \mu \mathrm{m}$ en adultos, tuis ( 2 años) y crías de alpacas recién llegadas a Nueva Zelanda, respectivamente. 
Cuadro 3. Correlaciones entre las principales características de la fibra de alpacas de las comunidades del distrito de Cotaruse, Apurímac (Perú)

\begin{tabular}{llll}
\hline & MDF & CV(DF) & IC \\
\hline $\mathrm{CV}$ & $-0.13^{\mathrm{ns}}$ & & \\
$\mathrm{CF}$ & $-0.99^{* * *}$ & $0.01^{\mathrm{ns}}$ & \\
$\mathrm{FC}$ & $-0.61^{* * *}$ & $-0.15^{\text {ns }}$ & $0.62^{* * *}$ \\
\hline
\end{tabular}

MDF: media del diámetro de la fibra; $C V(D F)$ : coeficiente de variación del diámetro de la fibra; FC: factor de confort; IC: índice de curvatura; ns: no significativo; ${ }^{* * *}: p<0.001$

El coeficiente de variación CV(DF) no tuvo un comportamiento definido, pero disminuyó ligeramente con la edad del animal. El FC disminuyó consistentemente a medida que aumentó la edad (desde $92.38 \pm 4.42 \%$ al año hasta $86.45 \pm 3.21 \%$ a los 4 años o más de edad), mientras que el IC no presentó un comportamiento regular con la edad, donde el mayor valor se observó a los dos años $(40.19 \%)$ y el menor valor al año de edad (33.35\%). McGregor y Butler (2004) en su estudio sobre las fuentes de diámetro de la fibra concluyeron que «los datos indican que el momento óptimo para tomar la muestra de fibra de alpaca es de aproximadamente dos años de edad». Sin embargo, los datos encontrados en este estudio y otros apoyan la idea de que la evaluación de la finura de la fibra en la alpaca se podría realizar con éxito al año de edad del animal. Dado que la edad es una variable importante, especialmente para los programas de mejoramiento genético, se requiere definir la edad más adecuada para evaluar la calidad de la fibra en la alpaca.

\section{Efecto del Sexo}

La MDF presentó una diferencia de $1 \mu \mathrm{m}$ por efecto de sexo a favor de las hembras $(\mathrm{p}<0.05)$. El CV(DF) fue similar para ambos sexos y el FC e IC fueron ligeramente mayo- res en las hembras $(p<0.05)$. Los valores de la MDF concuerdan con los hallazgos de Montes et al. (2008) quienes encontraron que las fibras de las hembras presentan $1 \mu \mathrm{m}$ de diámetro menor que las de los machos. Diferencias menores $(0.4-0.5 \mu \mathrm{m})$ fueron reportadas por Lupton et al. (2006) y Vásquez et al. (2015) a favor de las hembras. En contraste, hay informes que indican que no existen diferencias significativas por efecto del sexo en la MDF (McGregor y Butler, 2004; Huanca et al., 2007; Ormachea et al., 2015).

\section{Efecto del Color del Vellón}

El color del vellón presentó un efecto fuerte y consistente, ya que la fibra blanca exhibe mayor finura que las fibras de colores intermedios y oscuros. La diferencia entre la MDF del vellón blanco con el de color intermedio fue de $1.51 \mu \mathrm{m}$ y con el color obscuro de $4.39 \mu \mathrm{m}$. Por otro lado, no se observó diferencia significativa por efecto del color del vellón en el CV(DF).

El FC y el IC mostraron disminuciones consistentes, aunque en pequeñas proporciones, a medida que el color va del blanco a oscuro (FC: $92.16,87.89,75.94 \%$ e IC: 38.29 , $\left.34.98,29.26^{\circ} / \mathrm{mm}\right)$.

Similares diferencias de MDF fueron reportadas en alpacas de Nueva Zelanda (McGregor y Butler, 2004). Lupton et al. (2006), asimismo, indican que las fibras blancas eran más finas $(25.0 \mu \mathrm{m})$ que las de color negro $(29.5 \mu \mathrm{m})$, marrón $(26.1 \mu \mathrm{m})$ y gris plateado $(28.5 \mu \mathrm{m})$. Apoyado en estos resultados, se puede afirmar que la fibra de las alpacas blancas tiene una mayor finura de vellón, lo cual es probablemente debido al efecto de la selección de alpacas blancas que se ha practicado durante décadas en la zona sur del Perú para el color blanco.

\section{Efecto de la Comunidad}

Se pueden esperar diferencias en la calidad de la fibra de alpaca según el lugar de procedencia, dado que cada localidad o región tiene sus propias características. Los 
Cuadro 4. Categorías de las fibras de alpaca de las comunidades del distrito de Cotaruse, Apurímac (Perú), según la Norma Técnica Peruana NTP N. ${ }^{\circ} 231.301: 2004$

\begin{tabular}{lcc}
\hline Categoría & $\begin{array}{c}\text { NTP } \\
(\mu \mathrm{m})\end{array}$ & $\begin{array}{c}\text { Fibra } \\
(\%)\end{array}$ \\
\hline Baby & $<23.0$ & 53.10 \\
Fleece & $23.1-26.5$ & 26.90 \\
Medium & $26.6-29.0$ & 11.03 \\
$\quad$ fleece & & \\
Huarizo & $29.0-31.5$ & 7.59 \\
Grueso & $>31.5$ & 1.38 \\
\hline
\end{tabular}

resultados se pueden agrupar, convencionalmente, en tres grupos para establecer diferencias según los valores de la MDF y $\mathrm{CV}(\mathrm{DF})$. Así, Iscahuaca es la comunidad que posee la fibra más fina $(21.90 \mu \mathrm{m})$, seguida por el grupo de Pilluni y Quilcaccasa, que tiene poco más de $1 \mu \mathrm{m}$ sobre el valor de la primera, y el tercer grupo constituido por Pampamarca y San Miguel de Mestizas, cuyo valor de MDF es más de $2 \mu \mathrm{m}$ con relación al valor del primer grupo.

Estos resultados podrían explicarse si se considera que las primeras comunidades, especialmente Iscahuaca, han tenido el interés de seleccionar a las alpacas en función de finura y, tal vez, han recibido un mayor apoyo para la introducción de mejores machos para la fibra de calidad blanca. Montes et al. (2008) en Huancavelica encontró una diferencia significativa de $3.5 \mu \mathrm{m}$ entre ocho comunidades; sin embargo, Ormachea et al. (2015) y Huanca et al. (2007), ambos en Puno, no encontraron diferencias entre comunidades, aún al comparar comunidades ubicadas en diferentes zonas ecológicas (Puna seca y Puna húmeda).

Los valores de CV, FC e IC muestran pequeñas, aunque significativas diferencias entre comunidades. Así el CV(DF) varió entre 21.33 y $23.11 \%$, el FC entre 84.20 y $95.86 \%$ y el IC entre 31.43 y $43.07^{\circ} / \mathrm{mm}$.

\section{Efecto del Sitio de Muestreo}

Las diferencias de la MDF atribuibles al sitio de muestreo de la fibra no fueron significativas. El costillar medio presentó la menor MDF $(22.90 \mu \mathrm{m})$, habiendo una diferencia de 0.11 y $0.81 \mu \mathrm{m}$ para las muestras de la grupa y la paleta, respectivamente, así como una escasa variabilidad CV(DF). Los resultados sugieren que la zona del costillar podría, en todo caso, ser el sitio más apropiado para el muestreo de los vellones.

Newman y Peterson (1996) y Wuliji et al. (2000), en estudios sobre el rendimiento de la producción de alpaca en Nueva Zelanda, concluyeron que el costillar medio es la mejor zona corporal para el muestreo porque presentaba una alta correlación con muestras de otros sitios del cuerpo. No obstante, Aylan-Parker y McGregor (2002) concluyeron que «si se van a seleccionar alpacas para características tales como MDF baja y alto peso de vellón, se recomienda el sitio de costillar medio para el muestreo; pero si las alpacas son seleccionadas para un bajo coeficiente de variación MDF, baja incidencia de fibras meduladas y otras características de la fibra medulada, entonces se recomienda el muestreo en rejilla (saddle grid en inglés) como la técnica de muestreo óptimo». McGregor et al. (2012) estudiando la variación de la fibra en varios sitios del cuerpo de alpaca, encontraron que la muestra del sitio posterior del vellón (VP) tenía mejores correlaciones con muestras de las otras partes del cuerpo que las correlaciones de la MDF del costillar medio. Tomando estas diferencias y considerando que el sitio de muestreo es la variable de importancia suprema para su aplicación práctica, se requiere de estudios adicionales para definir la zona corporal más apropiada para determinar la calidad del vellón total en el cuerpo de alpaca. 


\section{Conclusiones}

- La evaluación de la calidad de la fibra de alpaca podría realizarse al año de edad, aunque la zona corporal más apropiada no pudo ser plenamente definida.

- La calidad de la fibra está fuertemente influenciada por la edad, el color del vellón y la comunidad de procedencia de los animales, y con menos influencia por el sexo y la zona corporal de la toma de la muestra.

- Las alpacas del distrito de Cotaruse producen fibra con buena finura que satisface las exigencias de la industria textil; además, la población actual tiene un gran potencial de variabilidad para el mejoramiento genético.

\section{Literatura Citada}

1. Aylan-Parker J, McGregor BA. 2002. Optimising sampling techniques and estimating sampling variance of fleece quality attributes in alpacas. Small Ruminant Res 44: 53-64. doi: 10.1016/ S0921-4488(02)00038-X

2. Bustinza AV. 2001. La alpaca. Conocimiento del gran potencial andino. Puno: Oficina de Recursos del Aprendizaje, Universidad Nacional del Altiplano. $495 \mathrm{p}$.

3. Germaná C, Chaquilla O, Santos G, Ferrari $M$, Krusich $C$, Kindgard $F$. 2016. Estudio socioeconómico de los pastores andinos de Perú, Ecuador, Bolivia y Argentina. Arequipa, Perú: El Alva. $537 \mathrm{p}$.

4. Gutierrez L. 2011. Las normas técnicas peruanas y su impacto en el desarrollo de los camélidos peruanos. Lima, Perú: Ministerio de Agricultura. [Internet]. Disponible en: http:// infoalpacas.com.pe/wp-content/uploads/ 2014/02/normastecnicasysuimpacto.pdf

5. Huanca T, Apaza N, Lazo A. 2007. Evaluación del diámetro de fibra en alpacas de las comunidades de los dis- tritos de Cojata y Santa Rosa - Puno. En: XX Reunión de la Asociación Latinoamericana de Producción Animal (ALPA). Cusco, Perú.

6. [INEI] Instituto Nacional Estadística e Informática. 2012. IV Censo Nacional Agropecuario 2012. Perú. [Internet]. Disponible en: http://censos.inei.gob.pe/ cenagro/tabulados/

7. Lupton CJ, McColl A, Stobart RH. 2006. Fiber characteristic of the Huacaya alpaca. Small Rumimant Res 64: 211-224. doi: 10.1016/j.smallrumres.2005.04 .023

8. McGregor BA. 2006. Production attributes and relative value of alpaca fleeces in southern Australia and implications for industry development. Small Ruminant Res 61: 93-111. doi: 10.1016/j.smallrumres.2005.07.001

9. McGregor BA, Butler KL. 2004. Sources of variation in fiber diameter attributes of Australian alpacas and implications for fleece evaluation and animal selection. Austral J Agric Res 55: 433-442. doi: 10.1071/AR03073

10. McGregor BA, Ramos HE, Quispe EC. 2012. Variation of fibre characteristics among sampling sites for Huacaya alpaca fleeces from the High Andes. Small Ruminant Res 102: 191196. doi: 10.1016/j.smallrumres.2011.07.016

11. Mellisho E. s/f. Producción de camélidos sudamericanos. Lima, Perú: Universidad Nacional Agraria. 26 p. [Internet]. Disponible en: http://infoalpacas.com.pe/wpcontent/uploads/2014/03/zoote-generalcamelidos222.pdf

12. Newman SAN, Paterson DJ. 1996. Variation in fleece characteristics over the body of alpaca. Proc NZ Soc Anim Prod 56: 338-341.

13. Montes M, Quicaño I, Quispe EC, Alfonso L. 2008. Características de la fibra de alpaca Huacaya producida en la región Altoandina de Huancavelica, Perú. En: Actualidad sobre adaptación, producción, reproducción y mejora genética en camélidos. Huancayo, Perú: Grafica Industrial. p 37- 47. 
14. Ormachea E, Calcín B, Olarte U. 2015. Características textiles de la fibra en alpaca Huacaya del distrito de Corani, Carabaya, Puno. Rev Investig Altoandinas 17: 215-220.

15. Quispe E. 2010. Evaluación de características productivas y textiles de la fibra de alpacas Huacaya de la región de Huancavelica, Perú. Simposio Internacional de Fibras de Camélidos Sudamericanos. Huancavelica, Perú.

16. $R$ Core Team. 2014. R: a language and environment for statistical computing. $\mathrm{R}$ Foundation for Statistical Computing. Vienna, Austria. [Internet]. Disponible en: http://www.R-project.org/

17. Tapia M, Flores J. 1984. Pastoreo y pastizales de los Andes del Sur. Progra- ma Cooperativo de Apoyo a la Investigación de Rumiantes Menores. Lima Perú: INIA. 321 p.

18. Vásquez O, Gómez OE, Quispe E. 2015. Características tecnológicas de la fibra blanca de alpaca Huacaya en la zona altoandina de Apurímac. Rev Inv Vet Perú 26: 213-222. doi: 10.15381/ rivep.v26i2.11020

19. Wuliji T, Davis GH, Dodds KG, Turner PR, Andrews RN, Bruce GD. 2000. Production performance, repeatability and heritability estimates for live weight, fleece weight and fiber characteristics of alpaca in New Zealand. Small Ruminant Res 37: 189-201. 